
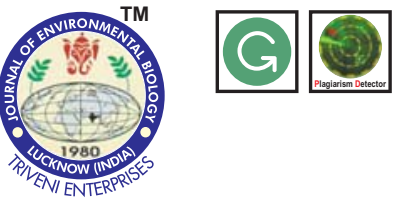

DOI: https://doi.org/10.22438/jeb/38/3/MS-184
Journal of Environmental Biology

ISSN: 0254-8704 (Print) ISSN: 2394-0379 (Online)

CODEN: JEBIDP

\title{
Studies on the effect of temperature variation on chromosomal changes in buckwheat (Fagopyrum esculentum Moench)
}

Authors Info

G. Kumar and A. Srivastava*

Department of Botany, University of Allahabad, Allahabad-211 002, India

*Corresponding Author Email : srivas.akanksha20@gmail.com

\section{Key words}

\section{Abnormality percentage,}

Active mitotic index,

Chromosomal aberrations,

Fagopyrum esculentum,

Temperature stress

\section{Publication Info}

Paper received : 12.08 .2015

Revised received : 08.01.2016

Re-revised received : 23.06 .2016

Accepted: 13.07 .2016

\section{Abstract}

Aim: Climatic fluctuations consequent into various kinds of environmental stresses. Among these, temperature stress is a major concerning factor with respect to plants. The aim of the present investigation was to study the effect of temperature stress on plant mitosis for different durations in the root meristems of Fagopyrum esculentum (buckwheat). Buckwheat is a sensitive plant with response to temperature stress.

Methodology: Exposure of cold and heat stress was given for 3, 5 and $7 \mathrm{hrs}$ to the root meristems of Fagopyrum esculentum, and their effects were studied at cytological level along with various aspects.

Results: Active mitotic index and chromosomal abnormalities were calculated that showed inverse relationship to each other. Different types of abnormalities were observed comprising scattering, c-mitosis, precocious movement, stickiness, unorientation, laggard formation, bridge formation etc., alongwith increasing duration of temperature.

Interpretation: After calculating active mitotic index (AMI \%) and abnormality percentages, it was concluded that heat stress was more effective as it showed various chromosomal anomalies in the root meristems of Fagopyrum esculentum as compared to cold stress.

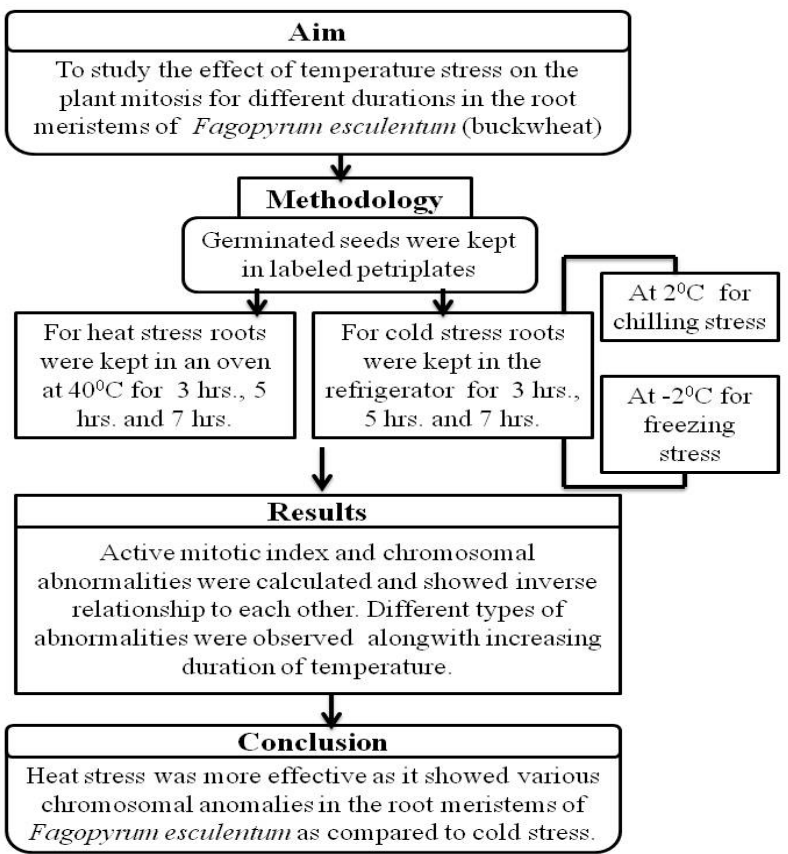




\section{Introduction}

Stress, the change of physiological conditions is caused by the factors that lead to disrupt the equilibrium of nature (Jaleel et al., 2009). The growth and productivity of plants are affected by environmental stress which alters their metabolic activities. So, environmental stress (abiotic and biotic) is the major aspect of consideration. Disparity of abiotic stress causes two types of effects in the environment viz., primary and secondary effects. Primary effect includes reduced water potential and cellular dehydration that directly changes the physical and biochemical properties of cells, further secondary effects reduces metabolic activity, ion toxicity and accelerates disruption of cellular integrity which may eventually lead to cell death (Vince and zoltan, 2011). Temperature stress comes under abiotic stress which affects the environment up to threshold level or lowers threshold level altering various developmental stages of plants which may proceed or not proceed for a longer term duration depending upon the exposure of temperature. Temperature stress also affects the enzyme activity and hence, can also affect mitosis because DNA replication, cell division and mitosis are controlled by an array of proteins and enzymes (Pathwayz: Factor that affects mitosis). These important events are highly regulated processes that are governed by specific genes within the cell.

Generally, high temperature is a critical factor for the growth of plants as compared to cold stress due to various reasonable factors. It also depends upon the tolerance of the plant, whether the climatic condition of plant is in favour of high or low temperature. Extreme temperature generate oxidative stress due to formation of reactive oxygen species (ROS). These ROS have a very detrimental effect on the biomolecular organization viz., DNA, proteins and lipid peroxidation (Apel and Hirt, 2004; Xu etal., 2006; Hasanuzzaman et al., 2012).

Generally, temperature between 25 to $30^{\circ} \mathrm{C}$ is considered optimum for growing crop plants. It varies from plant to plant and region to region. Temperature $40^{\circ} \mathrm{C}$ or above is called as a critical temperature for heat sensitive plant or non- tolerating plant (Wahid et al., 2007). Undesirable changes in plants due to increase in duration of temperature may cause heavy loss in crop production.

Cold stress is another environmental factor for affecting the plant growth and production causing damage to crops. Mainly two types of stresses come under the cold stress which involve chilling $\left(0-15^{\circ} \mathrm{C}\right)$ and freezing temperature $\left(>0{ }^{\circ} \mathrm{C}\right)$ (Miura and Furumoto, 2013). Chilling temperature is not more dangerous in contrast to freezing temperature. Freezing temperature also includes frost condition which ceases elongation of stem, inhibition of flowering, cell division, photosynthesis and affected metabolism. Freezing temperature is categorized into intracellular freezing which is a more serious threat because it occurs suddenly when ice usually formed in cell wall and intracellular spaces and damage occurs when ice crystals grow and perforate into the cytoplasm and second type of freezing is intercellular, not very effective and it occurs gradually but intracellular freezing (Stress Management: Agriculture).

Buckwheat (kuttu) is a pseudocereal and belongs to family Polygonaceae. The seeds are edible and rich in complex carbohydrates. It has diploid chromosome number $(2 n=16)$. It is very sensitive plant in response to environmental stresses, especially abiotic stresses such as temperature, drought and water stress. It is a low temperature enduring plant. Testing of plant root is significant as temperature fluctuations are conveniently assessed to the plant via root tip cells.

In view of the above, the present research was carried out to study cytological variations in root meristem cells of buckwheat due to temperature stress.

\section{Materials and Methods}

Plant material : The germplasm of Fagopyrum esculentum (Variety- VL-7) was obtained from National Bureau of Plant Genetic Resources, Shimla, Phagli for cytological analysis.

Germination of seed : The seeds were presoaked in a petridish using filter paper for the germination of root tips and kept into an incubator for $2-3$ days at $22-25^{\circ} \mathrm{C}$.

Heat and cold stress treatment: Germinated seeds were kept in labeled petriplates in an oven at $40{ }^{\circ} \mathrm{C}$ for heat stress and for cold stress roots were kept in the refrigerator at $2{ }^{\circ} \mathrm{C}$ (for chilling stress) and at $-2^{\circ} \mathrm{C}$ (for freezing stress) for different durations (3 hrs, $5 \mathrm{hrs}$ and $7 \mathrm{hrs}$ ) and one set was kept as control at room temperature. However, in freezing stress the roots were severely damaged even at lowest duration and hence, could not be cytologically analyzed.

Post treatment heat and chilling stress subjected sets were washed thoroughly in distilled water and fixed in carnoy's fixative for $24 \mathrm{hrs}$ and preserved in $90 \%$ alcohol for cytological study.

Cytological analysis : Root tips were hydrolyzed in $1 \mathrm{~N} \mathrm{HCl}$ for $2-$ 3 min. for softening and then stained with $2 \%$ acetocarmine for 30 min. Finally, slides were prepared by using squash technique for studying chromosomal and mitotic frequency. Chromosomal and mitotic aberrations were observed under light microscope at $40 \mathrm{X}$ resolution and microphotographs were taken by PCTV Vision Photography Software.

Active mitotic index (Edgar, 1961and Balong 1982) and abnormality percentage were calculated by the following formula:

$$
\begin{aligned}
& \text { Active mitotic index }=\text { Total no. of dividing cell } \\
& \text { (AMI) \% Total no. of cell observed } \times 100
\end{aligned}
$$


Total abnormality percentage $=$ No. of Abnormal cell

(TAB) \% $\quad$ Total no. of cell observed

Statistical analysis : For statistical analysis, three replicates for each treatment was used. Statistical analysis was performed by SPSS 16.0 software. One way analysis of variance (ANOVA) and Duncan's multiple range test (DMRT, $P<0.05)$ were conducted for mean separation and the graph was plotted by using sigma plot 10.0 software. Actual mean and standard error were calculated.

\section{Results and Discussion}

Buckwheat plant has 16 chromosomes in diploid set $(2 n=$ 16). The cytological observations displayed active mitotic index of $12.163 \%$ in control sets with normal pattern of chromosomes. At metaphase stage, 16 chromosomes were arranged at equatorial position (Fig. 1A) and anaphasic division showed normal separation in the ratio of 16:16 (Fig. 1B). The treatment of root tip cells with cold and heat stress along with increased subsequent duration changed the behaviour of chromosomes and the range of active mitotic indices along with abnormality percentage.

Active mitotic index decreased with increased duration of temperature in both the stresses with respect to control. A decrease in mitotic index might be due to inhibition of DNA synthesis at $S$ phase (Sudhakar et al., 2001).The rate of chromosomal aberrations increased with increasing duration of temperature. In case of cold stress, the percentage of active mitotic index showed reduction from $11.28 \%$ to $8.006 \%$ and from $10.643 \%$ to $7.441 \%$ in case of heat stress (Table 1 ). It was also been observed that low duration of temperature exposure was not much critical for the cells and tissues of the plant because abnormalities might be recovered, but at higher exposure of temperature i.e. 7 hrs with respect to cold and heat stress, a severe damage to cells and tissues were observed due to which cell elongation and cell distortion had occurred. Under both the stress at higher exposure, the rate of chromosomal abnormality increased while active mitotic index decreased (TAB\%$8.597 \pm 0.158$, AMl\%-8.006 \pm 0.159$)$ in cold stress and (TAB\%$9.424 \pm 0.309, \mathrm{AMI} \%-7.441 \pm 0.345$ ) heat stress as shown in Table1. A considerable frequency of metaphasic and anaphasic abnormalities was induced by cold and heat stress, which showed gradual increase alongwith duration of temperature exposure (Fig. 2 and 3). Various chromosomal anomalies such as scattering (Fig. 1C), C-mitosis (Fig. 1D), precocious movement (Fig. 1E), stickiness at metaphase (Fig. 1F), unorientation at metaphase (Fig. 1G), forward movement at anaphase (Fig. 1.H), unorientation at anaphase (Fig. 1I), stickiness at anaphase (Fig. $1 \mathrm{~J}$ ), laggard formation (Fig. 1K), bridge formation (Fig. 1L) etc. were noted under both heat and cold stress and increased duration. Heat stress showed higher percentage of chromosomal aberrations as compared to cold stress.
Many researchers have also studied and worked on temperature stress particularly high temperature on cell division and chromosomal behaviour in plants including sunflower varieties, wheat varieties, barley, maize and Vicia faba and have reported that the high temperature exhibits various types of chromosomal anomalies such as disturbed metaphase, anaphasic bridge, lagging chromosome and sticky chromosomes (Mashkina et al., 2002, Abou-Deif et al., 2007, Badr et al., 1988, Signem et al., 2013).

Mitotic index is an important parameter for determining the growth rate of root cells and chromosomal aberrations are the index of changes in the chromosomal behaviour (Liu et al., 1992) and inverse relationship of active mitotic index and rate of chromosomal aberrations clearly indicates that high temperature stress is very harmful in response to plant at cytological level. Under stress condition, all the cellular components including chromosomes are affected. DNA undergoes slight disturbance and thus creates conformational changes in the chromosomal structure inducing chromosomal abnormalities (Singh, 2014). Heat stress directly affects the cell cycle due to which cell functioning, cell distortion, distortion of spindle fibres or inhibition of DNA synthesis occurs rapidly as compared to cold stress. Buckwheat is cool adapting plant so it has capability of cold tolerance at chilling treatment. It causes little disturbance at chromosomal level. However, frost condition $\left(0^{\circ} \mathrm{C}\right)$ is more critical because it ceases cell elongation and cell division which lead to plant death.

The chromosomal aberrations or anomalies are a good indicator of deviations in the normal mechanism of cell cycle (Dhulgande et al., 2015). Scattering is induced by loss of microtubules of spindle fibres which retards the movement of spindle fibres towards the opposite poles. C-mitosis is caused due to inactivation of spindle fibres which delays division of centromere (Mann, 1977). The precocious movement occurs due to disturbed homology for chromosomal pairing, disruption of spindle fibres or inactivation of spindle mechanism (Agarwal and Ansari, 2001).

Predominant abnormalities such as clumping and stickiness is characterized by clustering of chromosomes at any phase of cell cycle (Paul et al., 2013). Genetic and environmental factors may also be responsible for clumping and stickiness which may affect the functioning of cell cycle. Gaulden (1987) investigated that chromosomal stickiness originated from dysfunctioning of one or two types of specific non-histone proteins involving chromosomal organization which are very important for chromatid separation and segregation. The functioning of proteins may be altered by mutation in structural gene (Turkoglu, 2007). Laggards occur due to delayed process of chiasma terminalisation (Pagliarini, 1990) or due to stickiness of chromosomal end (Kaur and Grover, 1985) or failure of chromosomal movement following dys functioning of spindle 
Sthols

A

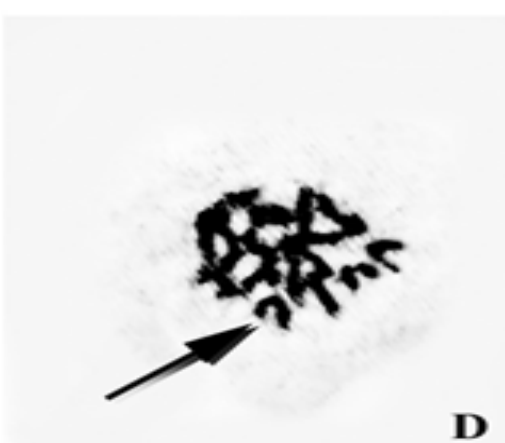

D

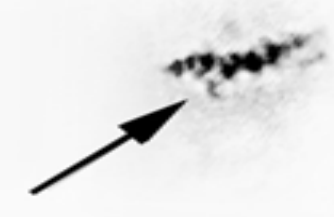

$\mathbf{E}$

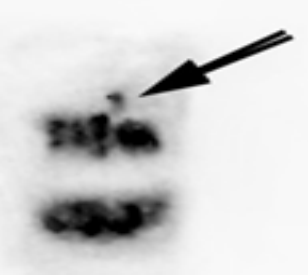

G

H

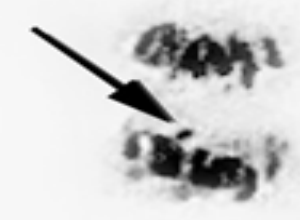

$\mathbf{J}$

$\mathbf{K}$

B
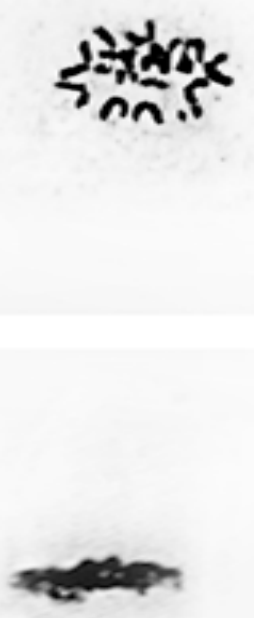

F

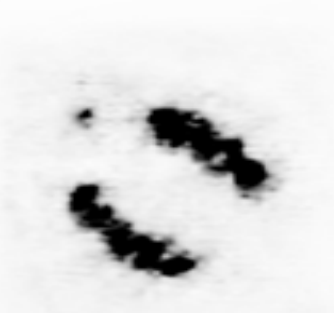

I

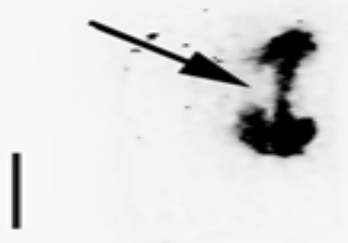

L

Fig. 1 : (A) Normal metaphase (2n= 16); (B) Normal anaphase (16:16 Separation); (C) Scattering at metaphase; (D) c-mitosis at metaphase; (E) Precocious movement at metaphase; (F) Stickiness at Metaphase; (G) Unorientation at metaphase; (H) Forward movement at anaphase; (I) Unorientation at anaphase; $(J)$ Stickiness at anaphase; $(K)$ Laggard formation at anaphase; (L) Single bridge formation at anaphase. [Scale bar: Length$6.42 \mu \mathrm{m}$ width- $7.5 \mu \mathrm{m}]$

fibres. At the time of pulling of chromosomes towards the poles, the bridges are broken into fragments which leads to the formation of laggards (Kumar and Tripathi, 2003). Apart from above abnormalities, a low frequency of bridge formation was also observed due to temperature stress on buckwheat. Bridges are formed due to breakage of chromosomes (Tomkins and Grant, 1972) or due to exchange of sister chromatids followed by failure of their segregation at later stages (Bharathi et al., 2014; 


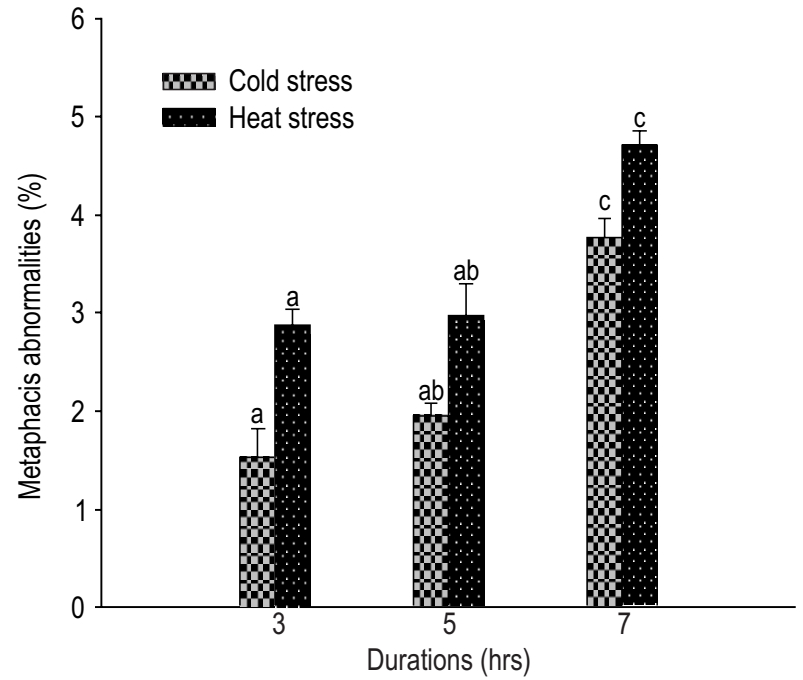

Fig. 2 : Metaphasic abnormalities induced by temperature stress in the root meristems of Fagopyrum esculentum moench

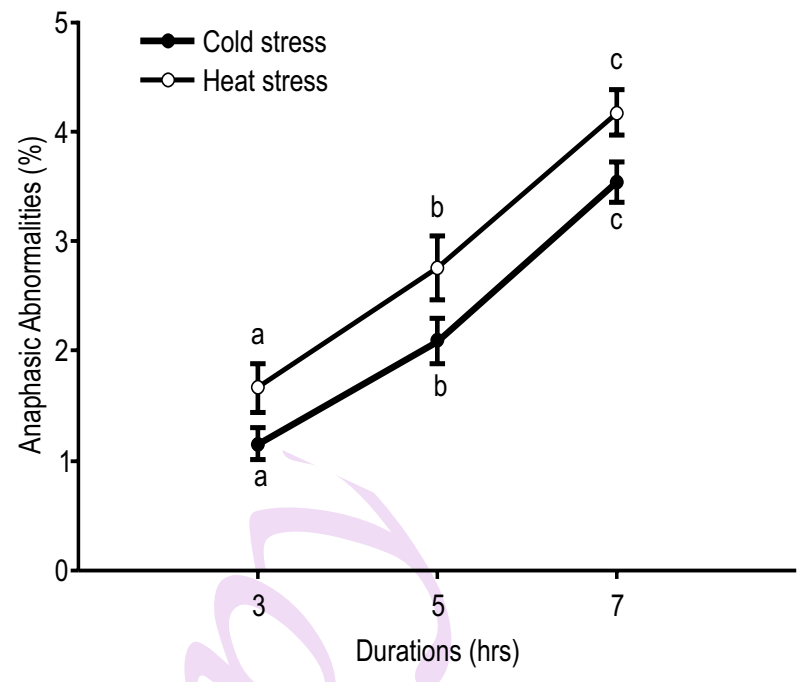

Fig. 3 : Anaphasic abnormalities induced by temperature stress in the root meristems of Fagopyrum esculentum Moench

Table 1 : A comparative account of cytological abnormalities induced by heat and cold stresses in root meristems of Fagopyrum esculentum Moench

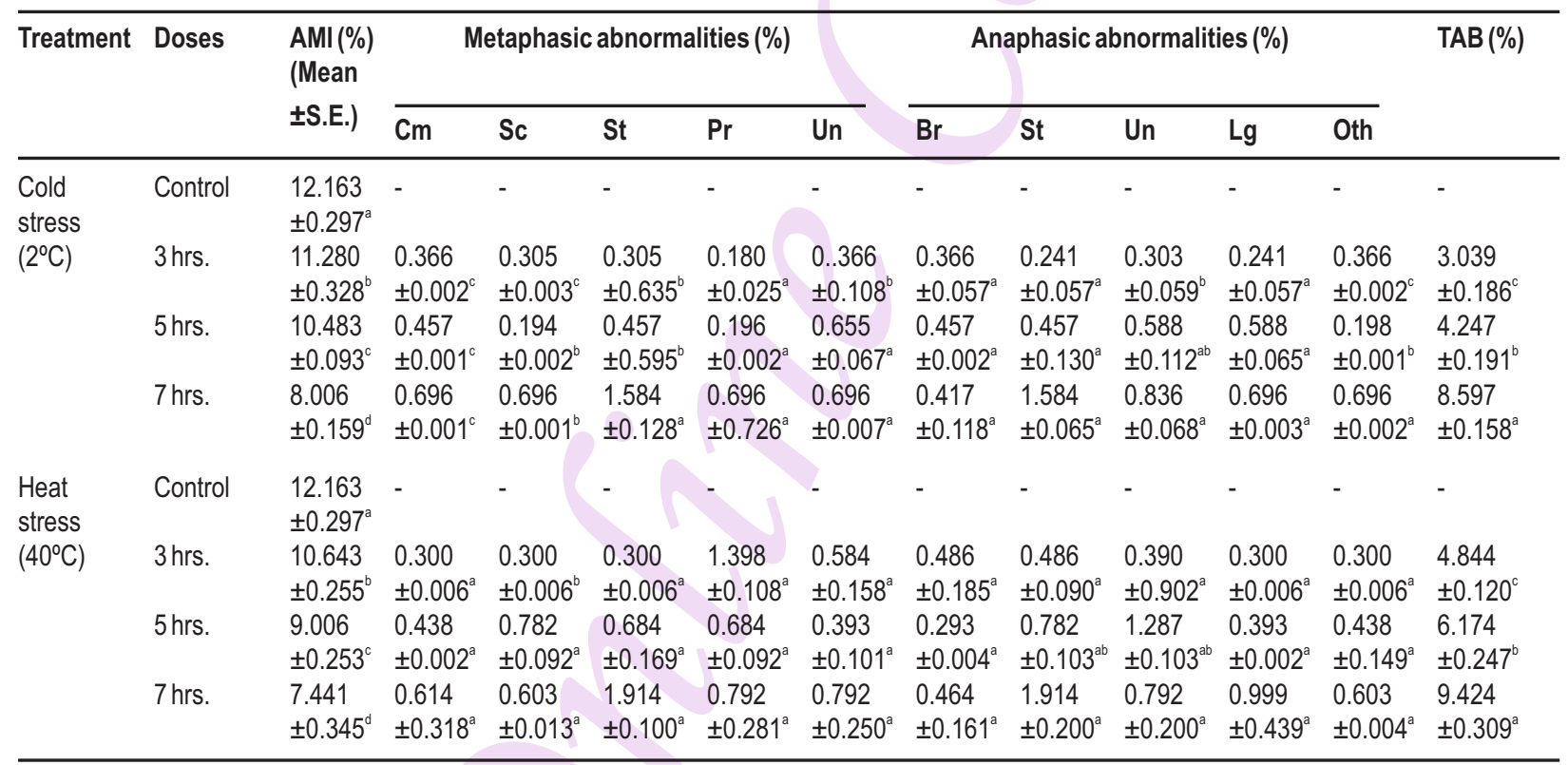

Where, Cm- C-Mitosis, Sc-Scattering, St-Stickiness, Pr-Precocious movement, Un- Un-orientation, Br-Bridge formation, Lg-Laggard formation, Fw- Forward movement, Oth-Others; Means followed by lowercase letter are statistically significant at $p<0.05$ in Duncan's Multiple Range Test; Values are \pm SE

Ahmad and Yasmin ,1992; Utsunomiya et al., 2002). Chromosomes are not able to move to opposite poles due to sticky nature resulting in bridge formation (Kumar and Rai, 2007). Bridges involves breakage and lateral fusion at same locus which leads to the formation of dicentric chromosomes (Rai and Kumar, 2010). The formation of dicentric and acentric chromosomes occur due to chromosomal inversion and these dicentric chromosomes are involved in bridge formation (Kumar and Dwivedi, 2013).Bridges are also formed due to opposite polarization of dicentric chromosomes (Anis et al., 1998). Chromosomal stickiness and attributed unequal translocation may also be responsible for bridge formation. Sticky bridge was also noticed in Fagopyrum esculentum induced temperature stress. The study assessed a basis for chromosomal aberrations 
under the influence of environmental stress. The outcome of this cytological study implicits that heat stress is more mitoclastic and mitotic decelerating in comparison to cold stress by analyzing the frequency of chromosomal aberrations which was more pronounced in heat stress. The study also signifies to check the tolerance of plants with respect to cold and heat stress.

\section{Acknowledgments}

The authors are grateful to the National Bureau of Plant Genetics Resources (NBPGR), Shimla, Phagli for providing inbred seeds and thankful to all lab members of Naithani Plant Genetics Laboratory for supporting and giving reinforcement for better presentation. The authors are also thankful to the Head of the Department for providing essential facilities for the experiments.

\section{References}

Abou-Deif, M.H. and F.I. Mohamed: Effect of heat stress on chromosomes and protein patterns in six hexaploid wheat varieties. Res. J. Cell. Mol. Biol., 1, 42-9 (2007).

Agarwal, R. and M.Y.K. Ansari: The effect of aniline on root tip cells of Vicia faba L. J. Cytol. Genet., 2, 129-134 (2001).

Ahmed, S. and R. Yasmin: Effect of methyl parathian and trimillox on the mitosis of Allium cepa. Cytologia., 57, 155-160 (1992).

Anis, M., B. Shiran and A.A. Wani: Genotoxic effect of aldrin and malathion on the root meristem of Vicia faba. Jour. Cytol. Genet., 33, 35-42 (1998).

Apel, K. and H. Hirt: Reactive oxygen species: metabolism, oxidative stress and signal transduction. Annu. Rev. Plant Biol., 55, 373-399 (2004).

Badr, A., M.A. Hamoud and A.R. El-Shanshouri: Effect of low and high temperatures on mitosis chromosomes in root meristem of barley and maize. J.K.A.U.: Educ. Sci., 1,69-74 (1988).

Balog, C.: The mitotic index in diploid and triploid in Allium roots. Cytologia, 47, pp. 689-697 (1982).

Bharathi, T., S. Gnanamurthy, D. Dhanavel and M. Ariraman: Induced physical mutagenesis and its effect in cytological behaviour of Ashwagandha (Withania somnifera (L.) Dunal). Inte. Letter Nat. Sci., 17, 152-161(2014).

Dhulgande, G.S., N. Jagtap, S. Parchande and S. Wagh: Impact of mutagenesis on cytological behaviour in chickpea (Cicer arietinum L.). Int. J. Curr. Microbiol. App. Sci., 2, 92-96 (2015).

Edgar, E.: Fluctuations in mitotic index in the shoot apex of Lonicera nitida. University of Canterbury, Christchurch, New Zealand, (1961).

Gaulden, M.E.: Some mutagens directly alter specific chromosomal proteins to produce chromosome stickiness. Mutagenesis, 2, 357$365(1987)$

Hasanuzzaman, M., M.A. Hossain, J.A. Teixeira da Silva and M. Fujita: Plant responses and tolerance to abiotic oxidative stress: Antioxidant defences is a key factors. Berlin: Springer, pp. 261-316 (2012).

Jaleel, C.A., P. Manivannan, A. Wahid, M. Farooq, R. Somasundaram, and R. Panneerselvam: Drought stress in plants: a review on morphological characteristics and pigments composition. Int. J.
Agric. Biol., 11, 100-105(2009).

Kaur, S. and I.S. Grover: Cytological effects of some organophosphorus pesticides. Cytologia, 50, 187-197 (1985).

Kumar, G. and P. Rai: Genotoxic potential of mercury and cadmium in soyabean. Turk. J. Biol., 31, pp.13-15(2007).

Kumar, G. and H. Dwivedi: Genotoxic effects of heavy metals in Trachyspermum ammi (L.) Sprague. Chromosome Bot., 8, 81-86 (2013).

Kumar, G. and A. Tripathi: Comparative mitotoxicity and mutagenecity of two heavy metals in Allium cepa L. J. Cytol. Genet., 4, 169-173 (2003).

Liu, D., W. Jiang and M. Li.: Effects of trivalent and hexavalent chromium on root growth and cell division of Allium cepa. Hereditas., 117, pp. 23-29 (1992).

Mann, S.K.: Cytological and genetical effects of dithane fungicides on Allium cepa. Environ. Experi. Bot., 17, 7-12 (1977).

Mashkina, E.V. and E.P. Gus'kov: Cytogenetic effect of temperature on the sunflower varieties. Tsitologiia, 44, 1220-1226 (2002).

Miura, K. and T. Furumoto: Cold signaling and cold response in plants. Int. J. Mol. Sci., 14, 5312-5337 (2013).

Pagliarini, M.S.: Meiotic behavior and pollen fertility in Aptenia cordifolia (Aizoaceae). Caryologia, 43, 157-162 (1990).

Pathwayz: Male AB Serum from FDA: Factor that affect mitosis. BranchGenetics.

Paul, A., S. Nag and K. Sinha: Cytological effects of Blitox on root mitosis of Allium cepa L. Int. J. Sci. Res. Publi., 3, 1-6 (2013).

Rai, P.K. and G. Kumar: The genotoxic potential of two heavy metals in inbred lines of maize (Zea mays L.). Turk. J. Bot., 34, 39-46 (2010).

Signem, O. and T. Selma: Cytogenetical and molecular responses of exogenous potassium sulphate for tolerance to extreme temperature in Vicia faba L. J. Pure App. Microbiol., 7, 663-670 (2013).

Singh, $\mathrm{V}$.: Effect of water stresses on microsporogenesis of a cultivated barley. Int. J.Advan. Res., 2, 110-115 (2014).

Stress Management: Temperature. Agriculture. Tnau. Agritech. portal.

Sudhakar, R., K.N.N. Gowda, and G. Venu: Mitotic abnormalities induced by silk dyeing industry effluents in the cells of Allium cepa. Cytologia, 66, pp. 235-239 (2001)

Tomkins, D.J. and W.F. Grant: Comparative cytological effects of the pesticides Menazon, Metrobromuron and TetrachloriosphthaIonitrile in Hordeum and Tradescantia. Can. J. Genet. Cytol., 14, 245-256 (1972).

Turkoglu, S.: Genotoxicity of five food preservatives tested on root tips of Allium cepa L. Mut. Res. Gen. Toxicol. Environ. Mutagen., 626, 414 (2006).

Utsunomiya, K.S., N.C.P. Bione and M.S. Pagliarini: How many different kinds of abnormalities could be found in unique endogamous maize plant? Cytologia, 67, 169-176 (2002).

Vince, O. and M. Zoltán: TAMOP 4.2.5 Book Database: Plant Physiology. Digitális Tankönvtár. (2011).

Wahid, A., I.S. Gelan, M. Ashraf and M.R. Foolad: Heat tolerance in plants: An overview. Environ. Experim. Bot., 61, 199-223 (2007)

Xu, S., J. Li, X. Zhang, H. Wei and L. Cui: Effects of heat acclimation pretreatment on changes of membrane lipid peroxidation, antioxidant metabolites, and ultrastructure of chloroplasts in two cool-season turf grass species under heat stress. Environ. Exp. Bot., 56, 274-285 (2006). 